\title{
Brain gangliosides in the presenile dementia of Pick
}

\author{
PE KAMP, WA DEN HARTOG JAGER, J MAATHUIS, PA DE GROOT, \\ JMBV DE JONG, PA BOLHUIS
}

From the Laboratory of Experimental Neurology, Department of Neurology, Academic Medical Centre, Amsterdam, The Netherlands

SUMMARY Histochemical analysis of frontal and temporal lobes from four patients with Pick presenile dementia indicated intracellular and extracellular deposits of gangliosides. Thin layer chromatography of gangliosides disclosed the presence of an unknown ganglioside, a decrease of $\mathrm{N}$-acetylgalactosamine- $G_{\mathrm{Dla}}$ and an increase of $G_{T l a}$ and/or $G_{D_{2}}$ in white matter of Pick brain. Chromatography of gray matter and quantitation of the sialic acid content yielded results similar to controls. It is suggested that degradation and removal of gangliosides is incomplete in Pick disease.

Pick presenile dementia or "progressive circumscribed cerebral atrophy" is clinically marked by a gradually progressive intellectual decline with decrease of comprehension, forgetfulness, aphasia, indifference to social customs, and lack of initiative. Anatomically, the most striking macroscopic feature in typical cases is a fronto-temporal atrophy with widely gaping sulci. The first histological description was provided in 1911 by Alzheimer. ${ }^{1}$

Intense loss of nerve cells is accompanied by cortical and subcortical gliosis, marked demyelination, and loss of axons. Distinctive is the appearance of swollen, rounded or pear-shaped cell bodies (the socalled ballooned cells) in which Bielschowsky's silver stain may disclose argentophilic Pick bodies. Senile plaques and neurofibrillary tangles are for the most part absent. The disease may be sporadic or autosomal dominant.

This paper reports a histochemical study indicating an abnormality of the gangliosides in Pick brain and subsequent analysis of these lipids by thin-layer chromatography.

\section{Patients}

Histochemistry was carried out on formalin-fixed brains from a brother (case 1) and a sister (case 2) with Pick disease who died at age 54 and 52 years respectively and on deep-

Address for reprint requests: Professor JMBV de Jong, MD, Department of Neurology, Academic Medical Centre, 1105 AZ Amsterdam, The Netherlands.

Received 27 November 1984 and in revised form 1 October 1985 Accepted 20 October 1985 frozen brains from a sporadic male case (case 3 ) and a familial male case (case 4) who died at age 57 and 47 years respectively.

Thin-layer chromatography of gangliosides was carried out in cases 1, 3 and 4. The father of cases 1 and 2 also died of Pick disease and was described by Schenk (case 8 in ref 2). Case 4 was member V-10 in a study of Groen and Endtz. ${ }^{3}$ The neuropathological diagnosis was established in all cases by the presence of frontal and temporal brain atrophy, loss of neurons, gliosis and ballooning of neurons. Pick bodies were found in cases 1,2 and 3. Histochemical controls were single cases of Krabbe disease, Tay-Sachs disease, multiple sclerosis, a colloid cyst in the third ventricle and $G_{M 1}$ gangliosidosis. Controls for thin-layer chromatography were six patients who died of extracranial causes and a case of Alzheimer disease.

\section{Methods}

\section{Histochemistry}

Frontal and temporal lobes were histologically examined by haematoxylin-eosin staining and by the techniques of Nissl, Klüver-Barrera, Loyez and Bielschowsky. ${ }^{4}$ Histochemical staining of frontal and temporal lobes was carried out with $\mathrm{NaOH}-O T A N$ (osmium tetroxide-alpha naphthylamine, (ref 5 , p.695), acid haematin (ref 5, p.689), $\mathrm{NaOH}$-acid haematin (ref. 6, p.55), cresylviolet-acetic acid (ref 7, p.585), oil red $O$ (ref 4, p.259), PAN (perchloric acid-naphtoquinone, (ref 8, p.39), Congo red (ref 4, p.391), copper rubeanic acid (ref 5, p.61), Alizarine red (ref 9, p.76), modified PAS with pretreatments (periodic acid-Schiff, ref 10) and BHPS (borohydride-periodate-Schiff after Roberts (ref 8, p.54) with pretreatments. Pretreatment with chloroform-methanol was for $18 \mathrm{~h}$ at room temperature, pretreatment with neuraminidase for $24 \mathrm{~h}$ at $37^{\circ} \mathrm{C}$ (enzyme from Vibrio cholerae and/or Clostridium perfringens) and pretreatment with 0.1 $\mathrm{M} \mathrm{H}_{2} \mathrm{SO}_{4}$ for $2 \mathrm{~h}$ at room temperature. In cases 3 and 4 
BHPS-staining was also carried out after pretreatment with diastase (pH6.0, $\left.1 \mathrm{~h}, 37^{\circ} \mathrm{C}\right)$. The modified PAS stainings of deep-frozen specimens were carried out after exposure to formalin vapour for $24 \mathrm{~h}$ at room temperature. Specimens of Pick brain and controls were studied under polarised light and by fluorescence microscopy with and without pretreatment in chloroform-methanol.

\section{Thin-layer chromatography}

Frontal grey $(400 \mathrm{mg})$ or white $(700 \mathrm{mg})$ matter was homogenised for $10 \mathrm{~min}$ with $3 \mathrm{ml}$ of chloroform-methanol $(1: 1, \mathrm{v}: \mathrm{v})$ in a Potter-Elvehjem mixer. The suspensions were spun down at $1400 \mathrm{rpm}$ and decanted. The precipitates were extracted with $3 \mathrm{ml}$ of chloroform-methanol-water (32:63:5, v:v:v). The supernatants were combined and further purified according to Folch ${ }^{11}$ by addition of $4.3 \mathrm{ml}$ chloroform and $2 \cdot 2 \mathrm{ml}$ water. After separation into a methanol-water layer containing most of the gangliosides and a chloroform layer containing the more hydrophobic lipids, the latter layer was reextracted with the theoretical upper phase. The methanolwater layers were then combined, and the sialic acid content was determined by Warren's thiobarbituric acid method. ${ }^{12}$ For qualitative lipid chromatography of Pick brain, reextraction appeared superfluous and was abandoned. Thin-layer chromatography plates were coated with silica gel 60, (Merck Darmstadt, Cat. no. 5641 or 5715). Plates were equilibrated with solvent system at $22 \pm 1{ }^{\circ} \mathrm{C}$. Following separation, plates were dried with hot air, sprayed with a fine mist of Svennerholm's resorcinol- $\mathrm{HCl}$ reagent, ${ }^{13}$ and baked face-down on a glass plate at $100 \pm 5^{\circ} \mathrm{C}$ for $30 \mathrm{~min}$. This procedure demonstrated gangliosides as purple bands.

For one-dimensional chromatography, $0 \cdot 2-0 \cdot 3 \mathrm{ml}$ of grey matter extract or $0.4-0.5 \mathrm{ml}$ of white matter extract were applied on plates no. 5641, and the ascending solvent system of Ando et al $^{14}$ was used, chloroform-methanol-aqueous $\mathrm{NH}_{4} \mathrm{OH} 2 \cdot 5 \mathrm{M}(60: 35: 8$, v:v:v). In preliminary experiments, other methods described by Harth et al $^{15}$ and Ando et $a l^{14}$ resulted in lower resolution of the gangliosides. Increase or decrease of the chloroform content resulted in better separation of the more hydrophobic or the more polar gangliosides respectively, but the area between $G_{D l a}$ and $G_{D l b}$ remained unaffected. Application of smaller samples led to a better resolution but the bands of the abnormal gangliosides (see Results) became less clear.

For two-dimensional chromatography, the methods of Ledeen $e t a l^{16}$ and Hunter ${ }^{17}$ were used. Samples of grey $(0.5 \mathrm{ml})$ and white $(0.7 \mathrm{ml})$ matter extract were applied. The technique of Ledeen et al ${ }^{16}$ employed no. 5715 plates and as solvents first chloroform-methanol-aqueous $\mathrm{KCl} 33 \mathrm{mM}$ (50:40:10, v:v:v) and second chloroform-methanol-aqueous $\mathrm{KCl} 33 \mathrm{mM}$ containing $\mathrm{NH}_{4} \mathrm{OH} 2 \cdot 5 \mathrm{M}$ (50:40:10, v:v:v). Hunter's technique ${ }^{17}$ required no. 5641 plates. Solvents were first chloroform-methanol-aqueous $\quad \mathrm{CaCl}_{2} \quad 1.35 \mathrm{mM}$ (55:35:10, v:v:v), and second chloroform-methanol-aqueous $\mathrm{KCl} 33 \mathrm{mM}$ (50:40:10, v:v:v). Ganglioside names were abbreviated after Svennerholm. ${ }^{18}$

\section{Results}

The histological characteristics of Pick disease ${ }^{1}$ were found in all four cases. An example is shown in fig 1. Histochemical stainings of frontal and temporal lobes

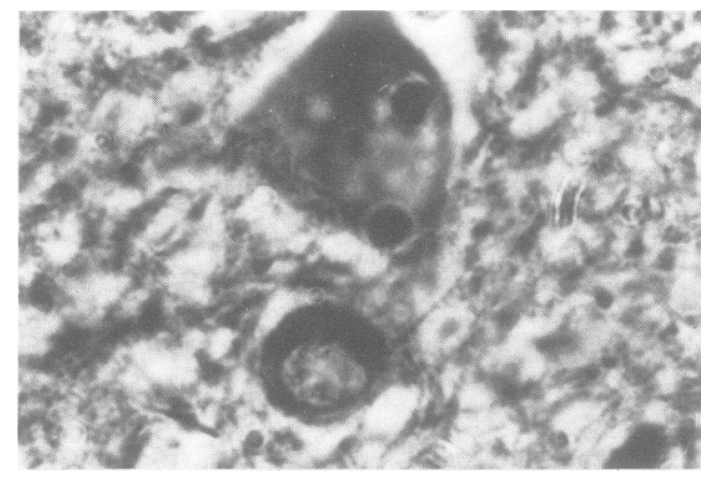

Fig 1 Ballooned cell with Pick inclusion body in temporal cortex of case 1. (Bielschowsky staining). ( × 1000)

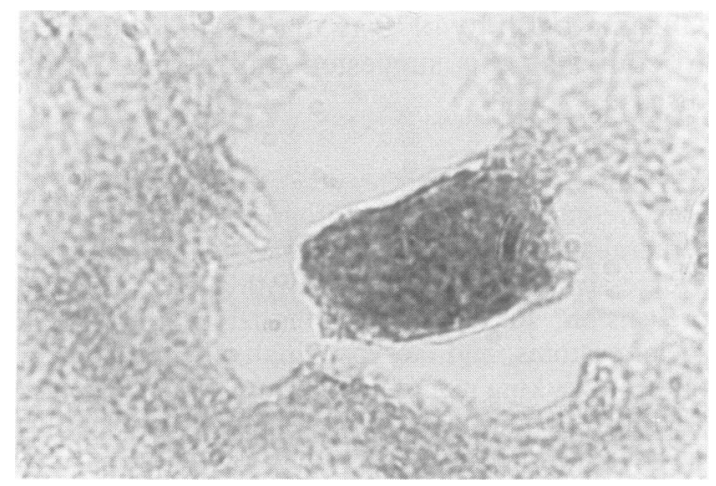

Fig 2 A modified-PAS positive neuron in temporal cortex of case $2 .(\times 1000)$

with $\mathrm{NaOH}-\mathrm{OTAN}$, acid haematin, $\mathrm{NaOH}$-acid hematin, PAN, Congo red and cresyl violet-acetic acid (see Methods) were similar to controls. With oil red $\mathbf{O}$ and Sudan black intracellular and extracellular deposits were visible in Pick brain. With Alizarine red $S$ only the extracellular deposits were seen. The modified-PAS and BHPS stainings demonstrated intracellular and extracellular gangliosides, as illustrated in figs $2-4$ and summarised in table 1 . The extracellular staining was abundant in white matter and sparse in grey matter. Diastase pretreatment (see Methods) had no effect.

By fluorescence microscopy a yellow-green autofluorescence was found in the neurons. In contrast to the modified-PAS and BHPS stainings, the autofluorescence remained after treatment with chloroform-methanol. Under polarised light, the deposits showed birefringence which disappeared by chloroform-methanol pretreatment.

By the modified-PAS method a similarly red staining was obtained in the ballooned cells of a case of 


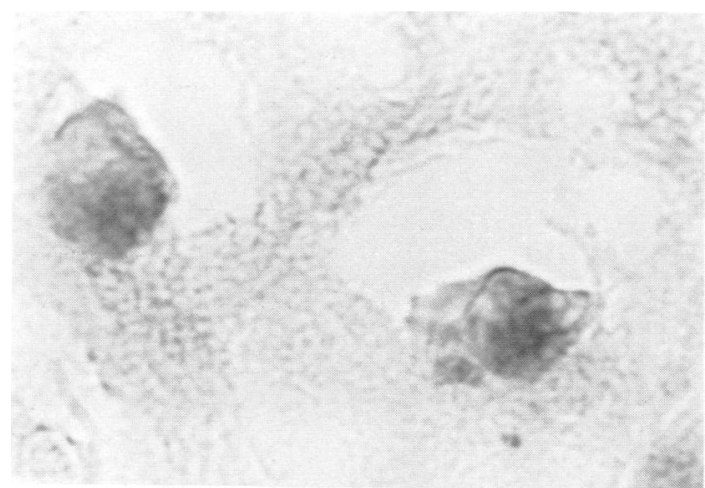

Fig 3 Two ballooned cells, modified-PAS positive in temporal cortex of case $2(\times 1000)$

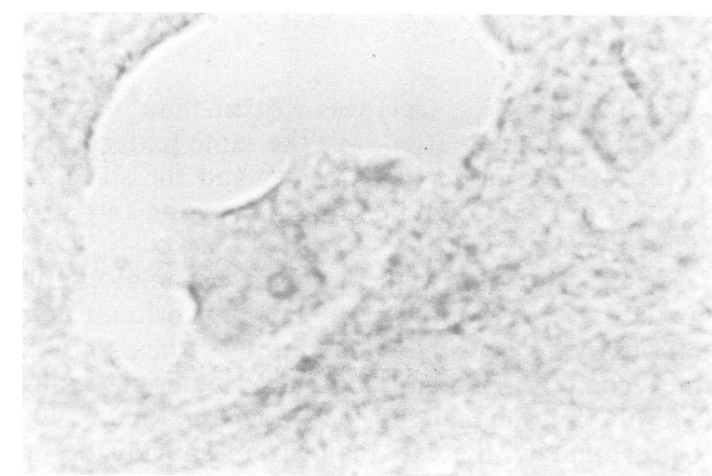

Fig 4 Modified-PAS staining after pretreatment with neuraminidase: the intracellular red colour seen in figs 2 and 3 is not found. Case 1, frontal cortex. $(\times 1000)$

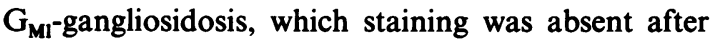
pretreatment by chloroform-methanol or neuraminidase. Globoid cells from a case of Krabbe disease (storage of cerebrosides) showed staining by the modified-PAS method which remained when a neuraminidase pretreatment was given but was absent after pretreatment with chloroform-methanol. The swollen cortical neurons in a case of Tay-Sachs disease showed staining by the modified-PAS and the BHPSmethod. The effect of pretreatment was similar to that

Table 1 Histochemical staining of Pick brain

\begin{tabular}{clll}
\hline Method & Pretreatment & $\begin{array}{l}\text { Staining } \\
\text { intracellular }\end{array}$ & $\begin{array}{l}\text { Staining } \\
\text { extracellular }\end{array}$ \\
\hline Modified & None & + & + \\
PAS & Chloroform-methanol & - & - \\
& Neuraminidase & - & + \\
BHPS & None & + & + \\
staining & Chloroform-methanol & - & - \\
& Neuraminidase & - & - \\
\hline
\end{tabular}

on the staining in Pick brain. Other controls showed no staining with the modified-PAS method.

\section{Thin-layer chromatography}

One-dimensional chromatography of frontal white matter from cases 1, 3 and 4 of Pick's disease disclosed two abnormalities in the area between $G_{D l a}$ and $G_{D l b}$ (fig 5, lane I, bands $A$ and $B$ ). In the control chromatograms (fig 5, lane II), band A was less pronounced, whereas B was absent. The ganglioside patterns of white matter from the Alzheimer and the control cases were alike. The chromatographic pattern of grey matter gangliosides was the same in control brain (fig 5, lane III), Pick disease (fig 5, lane IV), and Alzheimer's disease (not shown).

Two-dimensional chromatography of frontal white matter confirmed the differences between the ganglioside patterns in Pick disease and the control cases. Band A was resolved in two spots, designated A1 and A2. Figure 6 shows an example of the results obtained by the method of Ledeen et al: ${ }^{16}$ spot $\mathrm{Al}$ was weaker, spot A2 much stronger than in controls, which, furthermore, lacked a third spot B. These abnormalities were consistently found in three different samples of frontal white matter. They were demonstrated with equal efficacy by the methods of Ledeen $e t$ al $^{16}$ and Hunter. ${ }^{17}$ To exclude the possibility that the spots A2 and $B$ were lactone derivatives artificially formed during the procedure, we also changed the sequence of Ledeen and co-workers'16 developing systems. As pointed out by Hunter, ${ }^{17}$ the ammoniacal system would then reconvert the lactones to acids and artificial spots resulting from lactone formation would disappear. The spots A2 and B arose between $G_{D l a}$ and $G_{D l b}$ regardless of the separation sequence. Again, two-dimensional chromatography of Pick frontal grey matter yielded normal patterns.

The sialic acid determinations revealed no significant differences between the Pick and the control brains (table II).

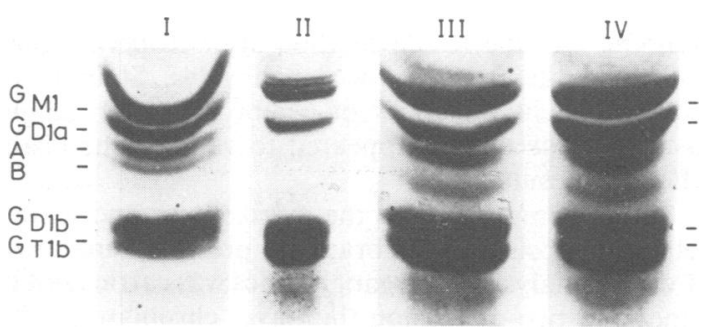

Fig 5 One-dimensional thin-layer chromatography of gangliosides as described by Ando et al. ${ }^{14} \mathrm{I}$ : Pick-white matter (case 1, frontal lobe); II: control-white matter; III: Pick-grey matter (case 1, frontal lobe); IV: control-grey matter. See text for bands $A$ and $B$. 

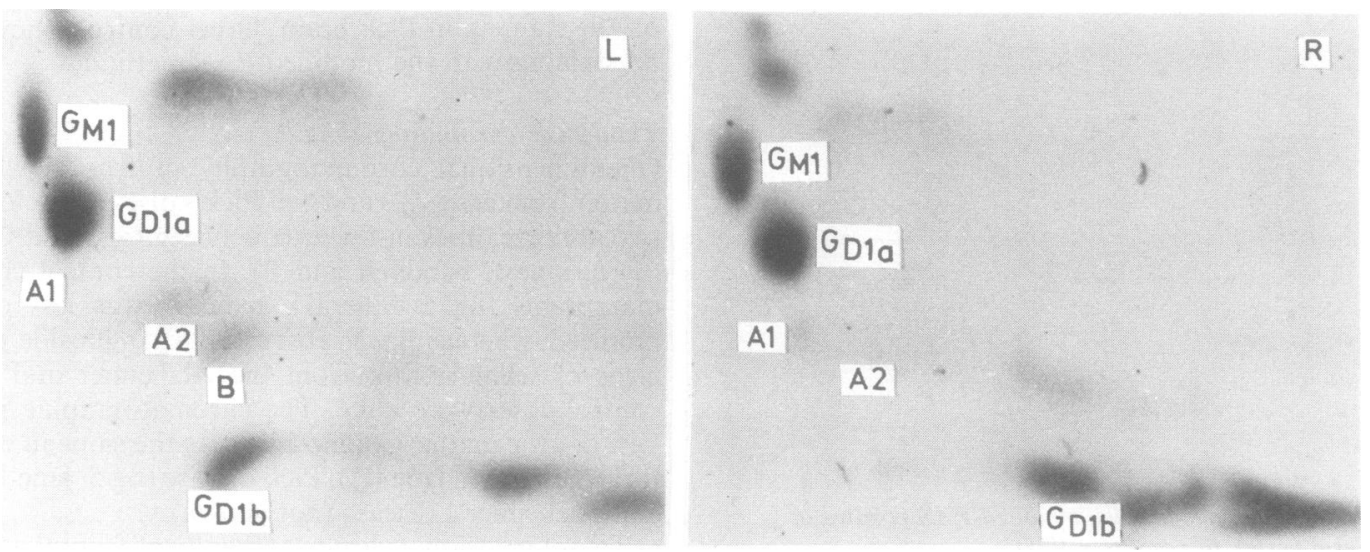

Fig 6 Two-dimensional thin-layer chromatography of gangliosides as described by Ledeen et al. ${ }^{16}$ Left: Pick-white matter (case 3, frontal lobe). The spot adjacent to $G_{\mathrm{M} 1}$ has not the characteristic purple colour of the gangliosides. Right: control-white matter. See text for spots $A 1, A 2$ and $B$.

Table 2 Sialic acid content of ganglioside extracts of brain tissue in micromole $100 \mathrm{~g}$ wet weight. SD between parentheses, $n$ is number of determinations.

\begin{tabular}{lcc}
\hline & Pick disease $(n=4)$ & Controls $(n=4)$ \\
\hline White matter & $98(26)$ & $80(8)$ \\
Grey matter & $207(60)$ & $187(58)$ \\
\hline
\end{tabular}

\section{Discussion}

Intracellular deposits in Pick brain have already been demonstrated with general lipid stainings by Jakob ${ }^{19}$ and by Roizin. ${ }^{20}$ With a large battery of more specific stainings (see Methods) we could not find abnormal accumulations of sphingomyelin, amyloid, phospholipids, triglyceride esters, cholesterol or cholesterol esters. Under polarised light birefringent crystals were visible and by fluorescence microscopy lipofuscin appeared present in the neurons. By Alizarine red staining calcium salts were found in extracellular (but not intracellular) storage products. More specific information was obtained by the modified-PAS staining and the BHPS method after Roberts which indicated the presence of gangliosides. Staining of controls including cases of cerebroside and ganglioside storage disease appeared to validate the histochemical results.

Other components of the intracellular and extracellular deposits in Pick brain are presently unknown. Further analysis of the gangliosides was carried out by one- and two-dimension thin-layer chromatography. The chromatographic results showed differences from normal ganglioside patterns: white matter contained a minor ganglioside absent in normals (B in figs 5 and 6), a spot representing $G_{T 1 a}$ and $G_{D 2}$ (A2) was stronger, and the spot representing $\mathrm{N}$-acetyl- galactosamine- $G_{D l a}(A 1)$ was weaker than in control specimens. Grey matter gave the same patterns as in controls. The major gangliosides and the sialic acid content of Pick brain did not differ significantly from control specimens.

The recent improvement of the chromatographic methods, for example the two-dimensional separations by Ledeen et $a l^{16}$ and Hunter, ${ }^{17}$ has simplified the recognition of details, but has also raised the number of separate ganglioside spots to about $120{ }^{17}$ Unfortunately, the vast majority of gangliosides has yet to be named. In our study, the identification of $\mathrm{N}$-acetylgalactosamine- $\mathrm{G}_{\mathrm{Dla}}$ and the mixture of $\mathrm{G}_{\mathrm{Tla}}$ and $G_{D 2}$ was made possible by the analytical work of Ando et $a l^{14}$ and Ledeen et al. ${ }^{16}$

The minor ganglioside B not observed in normals might be $\mathrm{N}$-acetylgalactosamine- $\mathrm{G}_{\mathrm{Tla}}$ as the change in retention of $B$ upon addition of ammonia was similar to that of $G_{D 3}, G_{D 2}$ and $G_{T 1 a}$. We have not yet been able to confirm this suggestion by pretreatment with $\mathrm{N}$-acetylgalactosaminidase.

In grey matter our chromatographic results did not show abnormalities in the gangliosides of three cases of Pick disease. As, by histochemistry, intracellular deposits of gangliosides were found, these gangliosides must have an abnormal localisation without a change in the total amounts of the various gangliosides. Op den Velde and Hooghwinkel found a decrease in $G_{D_{1 a}}$ and increased amounts of $G_{Q 1 b}, G_{M 3}$ and $G_{D l b}$ in a single case of Pick disease. ${ }^{21}$ Owing to the scarcity of Pick brain the significance of these discrepant results remains undecided.

Golgi studies of cortical neurons in Pick disease ${ }^{22}$ showed abnormal thinning of the dendritic arborisations. The resultant loss of neuronal surface would be expected to yield a decreased amount of gangli- 
osides; however, in the present study a normal amount was found. Therefore, and in view of the abnormal localisation, we suggest that the degradation and removal of gangliosides through the extra-cellular space is deficient in Pick disease.

We are grateful to Dr LJ Endtz (Westeinde Hospital, Den Haag), Dr S Stefanko (Erasmus University, Rotterdam) and Dr GThAM Bots (University of Leiden) for specimens of Pick brain used in this study, to Dr K Suzuki and Dr SU Walkley (Albert Einstein College, New York) for material from a case of

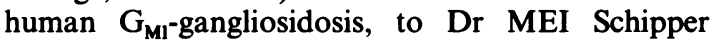
(Academic Medical Centre, Amsterdam) for control samples, to Mr BG Goldhoorn for sialic acid assays and to Ms A Kooyman and Ms SW Bosboom for secretarial assistance.

\section{References}

${ }^{1}$ Alzheimer A. Ueber eigenartige Krankheitsfälle des späteren Alters. Zbl ges Neurol Psychiat 1911;4:356-85.

${ }^{2}$ Schenk VWD. Maladie de Pick, étude anatomo-clinique de 8 cas. Ann Med Phys 1951;109:574-87.

${ }^{3}$ Groen JJ. Endtz LJ. Hereditary Pick's disease. Brain 1982;105:443-59.

${ }^{4}$ Romeis B. Mikroskopische Technik 16th ed. Munich Oldenbourg: 1968.

${ }^{5}$ Pearse AGE. Histochemistry, volume 1 London; Churchill, 1968.

${ }^{6}$ Adams CWM. Neurohistochemistry Amsterdam: Elsevier 1969.

${ }^{7}$ Lillie RD, Fullmer HM. Histopathology-Technic and Practical Histochemistry. New York: McGraw-Hill, 1976.

${ }^{8}$ High OB. Lipid Histochemistry, Oxford: University Press, 1984.

${ }^{9}$ Bancroft JD, Stevens A. Histopathological Stains and their Diagnostic Use. Edinburgh: Churchill Livingstone, 1975.

${ }^{10}$ Adams CWM, High OB. Lipid histochemistry. In: Glick D, Rosenbaum RM, eds. Techniques of Biochemical and
Biophysical Morphology, vol. 2. New York: Wiley 1975:99-156.

${ }^{11}$ Folch J, Lee MBL, Stanley GHS. A simple method for the isolation and purification of total lipids from animal tissues. J Biol Chem 1957;226:497-509.

12 Warren L. The thiobarbituric acid assay of sialic acids. $J$ Biol Chem 1959;234:1971-5.

${ }^{13}$ Svennerholm L. Quantitative estimation of sialic acids II. A colorimetric resorcinol-hydrochloric acid method. Biochim Biophys Acta 1957;24:604-11.

${ }^{14}$ Ando S, Chang N, Yu RK. High-performance thin-layer chromatography and densitometric determination of brain ganglioside compositions of several species. Anal Biochem 1978;89:437-50.

${ }^{15}$ Harth S, Dreyfus H, Urban PF, Mandel P. Direct thinlayer chromatography of gangliosides of a total lipid extract. Anal Biochem 1978;86:543-51.

${ }^{16}$ Ledeen RW, Haley JE, Skriwanek JA. Study of ganglioside patterns with two-dimensional thin-layer chromatography and radio-autography. Detection of new fucogangliosides and other minor species in rabbit brain. Anal Biochem 1981;112:135-42.

${ }^{17}$ Hunter GD, Wiegant VM, Dunn AJ. Interspecies comparison of brain ganglioside patterns studied by twodimensional thin-layer chromatography. $J$ Neurochem 1981;37:1025-31.

${ }^{18}$ Svennerholm L. Chromatographic separation of human brain gangliosides. J Neurochem 1963;10:623-31.

${ }^{19}$ Jakob H. Zur pathologischen Anatomie der Pickschen Krankheit. II. Mitteilung: Die feineren histologischen Veränderungen und die Frage der Lokalisation des Prozessbeginns am Neuron. Arch Psychiat Nervenkr 1961;202:540:68.

${ }^{20}$ Roizin L, Kaufman MA, Wharton R. Unusual histochemical and electron microscope findings in a cerebral biopsy of Pick's psychosis. J Neuropath Exp Neurol 1969;28:153-4.

${ }^{21}$ Op den Velde W, Hooghwinkel GJM. The brain ganglioside pattern in presenile and senile dementia. $J \mathbf{A m}$ Geriat Soc 1975;23:301-3.

${ }^{22}$ Wechsler AF, Verity MA. Pick's disease. A clinical, computed tomographic and histologic study with Golgi impregnation observations. Arch Neurol 1982;39: 287-90. 